\title{
Erweiterungen kommunaler Handlungsspielräume durch die Europäische Förderpolitik
}

Potenziale und Perspektiven untersucht an Fallbeispielen aus der Region Oldenburg

(Westniedersachsen)

\section{Enlargement of scope for municipal action by European subsidy policy}

Potentials and perspectives examined in case studies from the Oldenburg region (western Lower Saxony)

\section{Kurzfassung}

Der Beitrag geht der Frage nach, inwieweit die Fördermöglichkeiten der Europäischen Union den Kommunen erweiterte Gestaltungsspielräume eröffnen. Untersucht wurde dies am Beispiel der nordwestniedersächsischen Stadt Oldenburg. Gegenstand der Untersuchungen war eine Bestandsaufnahme aller EU-Projekte in der Stadt Oldenburg in den Jahren 1997-2002 und eine qualitative Analyse von sieben ausgewählten EU-Projekten. Die Studie kommt zu dem Ergebnis, dass ein kommunales Bemühen um EU-Fördermittel durchaus lohnenswert ist. Viele EU-Projekte in Oldenburg zielen auf typische kommunale Politik- und Handlungsfelder wie Stadtentwicklung, Wirtschaftsförderung, Integration oder Qualifizierung. Ein effektives kommunales EU-Engagement erfordert aber auch ein qualifiziertes und differenziertes Beratungsangebot.

\begin{abstract}
This paper examines the question of to what extent the subsidies available from the European Union provide a larger scope of action to local authorities. This was studied for the case of Oldenburg, a city in north-western Lower Saxony. The object of the studies was an inventory of all EU projects in the city of Oldenburg in the period from 1997 to 2002, and a quantitative analysis of seven selected EU projects. The study concludes that municipal efforts to obtain EU subsidies are well worthwhile. Many EU projects in Oldenburg target typical municipal fields of policy and action, such as urban development, promotion of economic development, social integration, and vocational qualifications. However, effective municipal involvement in obtaining EU funds also requires a qualified and differentiated advisory service.
\end{abstract}

\section{Einleitende Überlegungen und Fragestellung}

Es besteht kein Zweifel daran, dass die Kommunen von den Folgen der Europäischen Integration vielfältig betroffen sind. So haben z. B. die Wettbewerbsliberalisierungen unmittelbare Konsequenzen für die kommunale Strom-, Energie- oder Wasserversorgung oder die OPNV-Dienstleistungen. Auch die Wettbewerbsund Subventionskontrolle der EU schlägt u.a. im Bereich der Wirtschaftsförderung, der Infrastrukturpla- nung oder bei Grundstücksverkäufen voll auf die kommunale Ebene durch (vgl. Thränhardt 1999, S. 365). Gleichzeitig beklagen die deutschen Kommunen, dass sie die politische Willensbildung der EU nur sehr eingeschränkt beeinflussen können. Zwar sind Kommunalvertreter im Ausschuss der Regionen (AdR) präsent. Allerdings ist der AdR nur ein Beratungsgremium der EU-Kommission und des EU-Parlaments. Er hat eine 
relativ schwache Position. Darüber hinaus engagieren sich die deutschen Kommunen durch eine ausgeprägte Lobbyarbeit der kommunalen Spitzenverbände (vgl. Nutzenberger 2002, S. 223) oder im Rat der Gemeinden und Regionen Europas. Größere Kommunen und Regionen unterhalten oder planen bereits eigene Europabüros in Brüssel, z. B. Wien, Mailand, die Vertretung der bayrischen Kommunen oder die Region Stuttgart.

Das Verhältnis zwischen deutschen Kommunen und der Europäischen Union war vor etwa zwei Jahren auch Thema im Deutschen Bundestag. Im September 2000 startete die CDU-Fraktion eine Große Anfrage an die Bundesregierung. Darin wurde zunächst darauf verwiesen, dass sich die Europäische Union zu einer Entscheidungsebene entwickelt habe, die neben Ländern und Bund auch wesentliche Bereiche der kommunalen Selbstverwaltung beeinflusse. Im Hinblick auf die Sicherung der kommunalen Selbstverwaltungsgarantie fragte die CDU-Fraktion an: „In welchen Bereichen stärken und in welchen Bereichen belasten Maßnahmen der Europäischen Union das Recht der deutschen Kommunen, alle Angelegenheiten der örtlichen Gemeinschaft eigenverantwortlich zu regeln?“ (Deutscher Bundestag, Drucksache 14/4171, Sept. 2000). Die Bundesregierung verwies in ihrer Antwort vom März 2001 zum einen auf die Möglichkeiten der deutschen Kommunen, durch den Ausschuss der Regionen auf die politische Willensbildung der EU Einfluss zu nehmen. Zum anderen, so die Bundesregierung, zielten die EU- und EG-Rechtsetzungen nicht in erster Linie auf eine Belastung der kommunalen Selbstverwaltungen. Im Gegenteil: „Derartige Vorschriften eröffnen den Kommunen auch erweiterte Spielräume und Rechte. Ausdrücklich gestärkt wird kommunales Selbstverwaltungsrecht vor allem dort, wo Förderprogramme der EG den Kommunen Mittel bereitstellen und diesen dadurch Gestaltungsmöglichkeiten auf örtlicher Ebene eröffnen." (Deutscher Bundestag, Drucksache 14/5636, März 2001). Die Bundesregierung betont also die neuen Optionen, die sich den Kommunen durch die EU-Politik bieten. Sie geht davon aus, dass den deutschen Kommunen durch die EU-Förder- und Aktionsprogramme erweiterte Handlungsspielräume eröffnet werden.

Es stellt sich damit die Frage, wie deutsche Kommunen diese Gestaltungsmöglichkeiten umsetzen: Inwieweit erweitern EU-Fördermittel tatsächlich die kommunalen Handlungsspielräume? Welche finanziellen, qualifikatorischen oder institutionellen Potenziale sind auf kommunaler Ebene vorhanden, um EU-Mittel zu akquirieren und den Nutzen von EU-Projekten zu optimieren? Aus der Beantwortung dieser Fragen lassen sich erste Anhaltspunkte ableiten, welche Handlungsstrategien eine „kommunale EU-Politik" verfolgen müsste und welche Anforderungen an ein solches Politikfeld zu stellen sind.

Es geht um ein zentrales Themenfeld regionalwissenschaftlicher Forschung, nämlich den Stellenwert lokaler Handlungsspielräume bei der Gestaltung internationalisierter Wirtschaftsräume (vgl. Danielzyk, Oßenbrügge 1996, S. 101). Vielfach wird die Auffassung vertreten, dass der kommunalen und regionalen Handlungsebene trotz der wachsenden Dominanz globalisierter Rahmenbedingungen eine wirkungsvolle Bedeutung beigemessen werden muss. Das Globale bzw. die Globalisierung wird zum Handlungshintergrund. Das „Lokale“ bzw. „Regionale“ ist die eigentliche Handlungsebene, auf der die für die Regionalentwicklung bedeutsamen Entscheidungen getroffen werden (vgl. Aschauer 2000, S. 590). Insbesondere die Konzepte und Ansätze zur endogenen Regionalentwicklung unterstreichen die Handlungspotenziale und Gestaltungsspielräume kommunaler und lokaler Akteure und Institutionen (vgl. Butzin 2000, S. $153 \mathrm{ff}$; Rösch 2000, S. 163 ff.; Bathelt, Glückler 2000, S. 168 ff.; Foißner 2000, S. 304 ff.). Einen hervorgehobenen Stellenwert messen diese Entwicklungskonzepte dem soziokulturellen Potenzial einer Kommune oder Region zu. Dies wird begrifflich und konzeptionell durch regionale Identitäten und kreative Milieus (wahlweise auch „lernende Regionen“) zu erfassen versucht. Diese lokalen Identitäten und Milieus fördern vor allem dann die endogene Entwicklung, wenn sich zwischen Kommunalverwaltung, Unternehmen und Bevölkerung funktionierende Kooperations- und Kommunikationsformen etablieren (vgl. Aschauer 2000, S. 594 ff.). Damit kommt der Netzwerkansatz ins Spiel: Ein intaktes regionales Milieu zeichnet sich dadurch aus, dass die soziokulturellen, die politikbezogenen und die betrieblichen Netzwerke funktionieren (vgl. Rösch 2000, S. 165; Aschauer 2000, S. 593 f.). Die endogenen Entwicklungskonzepte stützen somit die These, dass ein Ausbau von Vernetzungs- und Kommunikationsstrukturen für eine regionale Entwicklung förderlich sind. Interkommunale Leistungsvergleiche liefern ebenfalls Belege dafür, dass in den politisch-strategischen Handlungsfeldern vor allem solche Kommunen erfolgreich sind, die das Zusammenwirken aller Beteiligten in einem Netzwerk am besten zu moderieren und $\mathrm{zu}$ organisieren vermögen (vgl. Adamaschek 2001, S. 505). Werden diese Überlegungen und Erkenntnisse mit der Fragestellung der vorliegenden Untersuchung verknüpft, so ist ein geschärfter Blick auf die kommunalen, EU-bezogenen Beratungs- und Unterstützungsnetzwerke zu richten. Dadurch kann möglicherweise ein wesentlicher Beitrag zur Klärung der Frage geleistet werden, inwieweit die EU-Regionalpolitik die kommunalen Handlungsspielräume erweitert. 


\section{Erste Annährungen}

an kommunale Handlungsspielräume: Voraussetzungen für eine Akquirierung und Nutzung von EU-Mitteln

Die Anforderungen an die kommunale Selbstverwaltung und Selbstverantwortung der Städte und Gemeinden sind in den vergangenen Jahren erheblich gestiegen. Das „neue Steuerungsmodell“ fordert von den Kommunen mehr Effizienz und Effektivität (vgl. Eser 2001 , S. 331 f; Stucke, Schöneich 1999, S. 420 ff.; Schnappauf 1999, S. 433 f.). Allerdings stellen Icks und Richter (1999, S. 9) fest, dass deutsche Kommunen das neue Steuerungsmodell unterschiedlich gut implementiert haben. Die Voraussetzungen für ein effektives und effizientes kommunales Handeln differieren in dieser Hinsicht also erheblich. Dies schlägt sich dann auch auf den Wettbewerb um EU-Fördermittel nieder. Schon längst lassen sich Polarisationseffekte beobachten, wonach aktionsfähige, gut verwaltete Kommunen EU-Mittel erhalten, weniger dynamische Kommunen dagegen leer ausgehen (Thränhardt 1999, S. 372 f.). Dieser Prozess wird durch eine EU-Förderpolitik forciert, die sich in den vergangenen Jahren stärker wettbewerbsorientiert entwickelte. Es gibt immer häufiger Fördermodelle, in denen lokale Akteursgruppen um EU-Fördermittel konkurrieren (vgl. Toepel 2000, S. 399).

Durch die veränderten organisatorisch-institutionellen Rahmenbedingungen sowie den wirtschaftlichen Strukturwandel in den 90er Jahren hat auch die kommunale Wirtschaftsförderung und Wirtschaftspolitik einen wesentlichen Wandel ihrer Aufgaben- und Arbeitsfelder erfahren. Neue Aufgaben und Aktivitäten kamen hinzu. Diese rekurrieren auf die Rolle der Wirtschaftsförderung als Vermittler und Moderator, Initiator und Controller von Entwicklungsprojekten sowie Dienstleister für kommunale Unternehmen (vgl. Grabow, Henckel 1999, S. 624). Dementsprechend stehen in vielen Kommunen die Wirtschaftsförderer bei der Akquirierung und Verwaltung von EU-Mitteln oftmals an exponierter Stelle. Vielfach sind sie es, die die EUFörderprogramme und -richtlinien durchforsten und nach Finanzierungsmöglichkeiten für kommunale Unternehmen oder Projekte suchen. Gleichzeitig informieren und beraten sie in erster Linie kommunale Unternehmen, Existenzgründer oder Unternehmensverbände bzw. wirtschaftsnahe Institutionen über EUFördermittel. Insbesondere die EU-Strukturfondsprogramme (oftmals in Verbindung mit den Förderungen aus der Gemeinschaftsaufgabe zur Verbesserung der regionalen Wirtschaftsstruktur), die Gemeinschaftsinitiativen sowie die Innovativen Maßnahmen werden über die kommunalen Wirtschaftsförderungseinrich- tungen oder andere öffentliche Dienststellen der Kommunen moderiert und bewirtschaftet.

Das Förderspektrum der Europäischen Union geht allerdings weit über den vorwiegend an ökonomischen Aspekten orientierten Aktionsradius der kommunalen Wirtschaftsförderung hinaus. Eine Reihe von EU-Förderprogrammen zielt zum Beispiel auf Schulen (z. B. die Programme Comenius oder Sokrates), Integration von Minderheiten oder Benachteiligten (im Rahmen von EQUAL, ESF/Ziel 2 oder INTERREG), Forschungsoder Kultureinrichtungen (wie Erasmus, Sokrates oder die Forschungsrahmenprogramme) sowie private bzw. ehrenamtliche Institutionen oder Verbände (beispielsweise in Form lokaler Aktionsgruppen bei LEADER oder INTERREG). Um solche EU-Förderpotenziale ebenfalls für kommunale Akteure und Projektträger verfügbar zu machen, haben einige Kommunen eigene EU-Koordinierungsstellen oder Europateams eingerichtet, die die EU-Fördermöglichkeiten systematisch auswerten, z. B. Frankfurt a. M., Köln, München (vgl. Thömmes, Saller 2002, S. 54 f.). In vielen Kommunen erfolgt mittlerweile auch ein Outsourcing dieser Aufgaben an private Consultingunternehmen. Auch Transfer- und Koordinierungsstellen an Hochschulen leisten zum Teil solche Dienste. Wenn die Kommunen keine EU-Koordinierungsstellen unterhalten bzw. beauftragen können, müssen sie auf Ansprechpartner bei den Bezirks- oder Landesregierungen zurückgreifen. Darüber hinaus hat die Europäische Union insbesondere im ländlichen Raum europaweit ca. 130 Informationsstellen eingerichtet, die sogenannten Carrefours. Diese sollen Bürger, öffentliche und private Einrichtungen über die EU-Fördermöglichkeiten informieren.

\section{Erweiterung kommunaler Handlungsspielräume durch EU-Förderprogramme: das Fallbeispiel Oldenburg}

\subsection{Bestandsaufnahme und Evaluation von EU-Projekten in der Stadt Oldenburg: Untersuchungsraum, Projekte, Ziele und methodisches Vorgehen}

Die Stadt Oldenburg ist Oberzentrum mit ca. 156000 Einwohnern im nordwestlichen Niedersachsen. Eine Untersuchung zur Beschäftigungsentwicklung in den westlichen Bundesländern hat ergeben, dass Oldenburg mit einer Zuwachsrate von ca. $35 \%$ bundesweit zu den Beschäftigungsgewinnern gehörte (vgl. Möller, Tassinopoulos 2000, S. 21). In Oldenburg hat die Bezirkregierung Weser-Ems ihren Sitz, ebenso das EUInformationsbüro Carrefour Niedersachsen/Weser- 
Ems. Im Amt für Wirtschaftsförderung wurde kürzlich eine EU-Koordinierungsstelle eingerichtet. Hier werden städtische EU-Projekte koordiniert sowie Informationen über die EU und europäische Förderprogramme erteilt (vgl. Langer 2002, S. 8).

Im Rahmen eines Kooperationsprojekts, an dem das Fachgebiet Geographie der Universität Osnabrück, der Carrefour Niedersachsen/Weser-Ems und das Amt für Wirtschaftsförderung der Stadt Oldenburg beteiligt waren, wurden die EU-Aktivitäten in der Stadt Oldenburg eingehend untersucht. Zum einen ist für den Zeitraum von 1997 bis 2002 eine systematische Bestandsaufnahme aller in der Stadt Oldenburg durchgeführten EU-Projekte vorgenommen worden. Neben dieser Bestandsaufnahme wurden zum anderen sieben abgeschlossene EU-Projekte ausgewählt und einer eingehenden Analyse und Evaluation unterzogen (vgl. Rolfes 2002, S. 15 f.).

\subsection{Bestandsaufnahme von EU-Projekten in Oldenburg von 1997 bis 2002}

Auf der Basis unterschiedlicher Quellen wurden alle laufenden und abgeschlossenen EU-Projekte in der Stadt Oldenburg zwischen 1997 und 2002 erhoben. Von den Förderprojekten wurden die Titel bzw. der Inhalt, das zugrundeliegende EU-Förderprogramm sowie der Projektträger oder Projektpartner in Oldenburg erhoben. Für die überwiegende Zahl der Förderprojekte konnten zusätzlich die in den Jahren 1997-2002 nach Oldenburg geflossenen Fördersummen ermittelt werden. Das Ziel dieser quantitativen Analyse war es, Transparenz über Ausmaß und Inhalte der EU-Förderprojekte in der Stadt zu erhalten. Zudem sollte die Bestandsaufnahme zeigen, in welchen kommunalen Aktionsfeldern die akquirierten EU-Fördermittel zusätzliche Handlungsspielräume geschaffen haben. Die Bestandsaufnahme fand unter maßgeblicher Mitwirkung des Carrefour Niedersachsen/Weser-Ems statt (vgl. die ausführliche Darstellung in Carrefour Niedersachsen/Weser-Ems 2002, S. 11 ff.).

In dem Beobachtungszeitraum wurden 215 Projekte von der Europäischen Union finanziell gefördert. Insgesamt waren 60 verschiedene öffentliche und private Einrichtungen sowie Unternehmen aus der Stadt Oldenburg als Projektträger oder Projektpartner an EU-Projekten beteiligt bzw. haben Fördermittel in Anspruch genommen. Gefördert wurden die Projekte aus unterschiedlichen EU-Programmen: den Strukturfondsprogrammen (Ziel 2 und 3), aus Gemeinschaftsinitiativen (EQUAL, ADAPT, KONVER, ...), innovativen Maßnahmen (LOS, RIS,...) oder anderen Förderprogrammen (4. und 5. Forschungsrahmenprogramm,
ERASMUS, LEOARDO,...). Nach überschlägigen Berechnungen sind zwischen 1997 und 2002 jährlich etwa 4 Mio. $€$ aus EU-Quellen nach Oldenburg geflossen

Bisher hat keine andere bundesdeutsche Kommune eine vergleichbare Bestandsaufnahme von EU-Projekten und akquirierten EU-Mitteln durchgeführt und veröffentlicht. Aufgrund mangelnder Vergleichsgrundlagen ist nicht abschätzbar, ob Oldenburg im interkommunalen Vergleich überdurchschnittlich oder unterdurchschnittlich abschneidet. Anhand des Datenmaterials kann aber geprüft werden, in welchen Förderbereichen die EU-Mittel akquiriert und eingesetzt wurden und welche Projektträger oder Projektpartner in Oldenburg aktiv waren. Die Tabelle 1 zeigt acht Förderbereiche, in die die EU-Projekte eingeteilt wurden.*

Tabelle 1

Anzahl und durchschnittliche Fördersummen der zwischen 1997 und 2002 in Oldenburg durchgeführten EU-Projekte (nach Förderbereichen)

\begin{tabular}{|c|c|c|c|}
\hline Förderbereiche & $\begin{array}{c}\text { Anzahl der } \\
\text { EU-Projekte }\end{array}$ & $\begin{array}{c}\text { davon mit } \\
\text { Angabe der } \\
\text { Fördersumme }\end{array}$ & $\begin{array}{c}\text { Fördersumme } \\
\text { je Projekt } \\
\text { (in e) }\end{array}$ \\
\hline Weiterbildung im Beruf & 16 & 3 & 38000 \\
\hline Schulische Bildung & 19 & 18 & 7000 \\
\hline $\begin{array}{l}\text { Berufliche Qualifizierung, } \\
\text { Integration, } \\
\text { Gesundheitsförderung }\end{array}$ & 45 & 38 & 14000 \\
\hline $\begin{array}{l}\text { Kultur- und } \\
\text { Jugendförderung }\end{array}$ & 10 & 10 & 50000 \\
\hline $\begin{array}{l}\text { Unterstützung der } \\
\text { regionalen Entwicklung }\end{array}$ & 6 & 6 & 66000 \\
\hline $\begin{array}{l}\text { Förderung von } \\
\text { Unternehmen oder } \\
\text { wirtschaftsnaher } \\
\text { Infrastruktur }\end{array}$ & 14 & 12 & 259000 \\
\hline $\begin{array}{l}\text { Kooperation und Mobilität } \\
\text { zwischen europäischen } \\
\text { Hochschulen }\end{array}$ & 16 & 10 & 103000 \\
\hline Forschungsförderung & 84 & 59 & 230000 \\
\hline
\end{tabular}

Quelle: Carrefour Nicdersachsen/Weser-Ems 2002 und eigene Berechnungen

Nach dieser Aufstellung hatten die Bereiche Forschungsförderung und Berufliche Qualifizierung, Integration und Gesundheitsförderung die meisten EUProjekte zu verzeichnen. Auch hinsichtlich des akquirierten Fördervolumens waren in diesen beiden Sektoren die höchsten Werte zu verzeichnen: Nach überschlägigen Berechnungen und Schätzungen wurde im Beobachtungszeitraum die Forschung in der Stadt Oldenburg mit ca. 13,5 Mio. $€$ gefördert. Bei diesen Förderprojekten (überwiegend im Rahmen des 
4. oder 5. Forschungsrahmenprogramms der EU) waren in der Regel keine monetären Komplementärfinanzierungen durch die Projektträger erforderlich. EU-Fördermittel für berufliche Qualifizierung, Integrationsmaßnahmen sowie Gesundheitsförderung wurden zwischen 1997 und 2002 in der Größenordnung von etwa 5,4 Mio. $€$ für Oldenburger Projektträger zur Verfügung gestellt. Für die Unterstützung von Unternehmen und die Förderung wirtschaftsnaher Infrastruktur flossen in den sechs Jahren etwas über 6 Mio. $€$ nach Oldenburg. Diese Mittel verteilen sich allerdings auf vergleichsweise wenige Projekte. Diese Projekte erforderten in der Regel komplementäre Mittel in mindestens denselben Größenordnungen. Werden für die einzelnen Förderbereiche die durchschnittlichen EU-Fördervolumina ermittelt, so verzeichnen die Forschungsförderung und die Förderung von Unternehmen sowie wirtschaftsnaher Infrastruktur mit durchschnittlichen EU-Zuschüssen von über 230000 bis $259000 €$ je Projekt die höchsten Werte (vgl. Tab. 1). Ebenfalls respektable Größenordnungen wurden für Kooperationen bzw. die Mobilität zwischen europäischen Hochschulen (vorwiegend im Rahmen des ERASMUS-Programms) erreicht. Erheblich geringer fallen die durchschnittlichen Projektkosten für die schulische Bildung sowie die berufliche Qualifizierung, Integration und Gesundheitsförderung aus.

Vergleichbare Zusammenstellungen sind auch für die Projektträger bzw. Projektpartner vorgenommen worden. Wie die Tabelle 2 ausweist, haben im Analysezeitraum Projektträger bzw. Projektpartner an Hochschulen und öffentlichen wie privaten Forschungsein-

Tabelle 2

Anzahl und durchschnittliche Fördersummen der zwischen 1997 und 2002 in Oldenburg durchgeführten EU-Projekte (nach Projektträgern bzw, -partnern)

\begin{tabular}{|l|c|c|c|}
\hline $\begin{array}{l}\text { Projektträger oder } \\
\text { Projektpartner }\end{array}$ & $\begin{array}{c}\text { Anzahl der } \\
\text { EU-Projekte }\end{array}$ & $\begin{array}{c}\text { davon mit } \\
\text { Angabe der } \\
\text { Fördersumme }\end{array}$ & $\begin{array}{c}\text { Fördersumme } \\
\text { je Projekt } \\
\text { (in e) }\end{array}$ \\
\hline $\begin{array}{l}\text { Hochschulen, } \\
\text { Forschungseinrichtungen } \\
\text { Kulturelle Vereinigungen } \\
\text { und Einrichtungen }\end{array}$ & 99 & 61 & 223000 \\
$\begin{array}{l}\text { Schulen } \\
\text { Soziale Vereinigungen } \\
\text { und Einrichtungen }\end{array}$ & 4 & 4 & 111000 \\
$\begin{array}{l}\text { Stadt Oldenburg } \\
\text { Unternehmen }\end{array}$ & 17 & 17 & 4000 \\
$\begin{array}{l}\text { Weiterbildungsträger, } \\
\text { Arbeitsamt }\end{array}$ & 19 & 14 & 174000 \\
Wirtschaftsnahe & 13 & 17 & 130000 \\
Vereinigungen und & 19 & 12 & 104000 \\
Institutionen & & & 73000 \\
\hline
\end{tabular}

Quelle: Carrefour Niedersachsen/Weser-Ems 2002 und eigene Berechnungen richtungen mit Abstand die meisten EU-Projekte durchgeführt oder waren daran beteiligt. Hinter dieser Vielzahl von Projekten verbergen sich neben den reinen Forschungsprojekten auch viele Kooperationsprojekte und Mobilitätsförderungen der Oldenburger Hochschulen. An zweiter Stelle befindet sich die Stadt Oldenburg. Von der Stadt wurden Projekte aus unterschiedlichen Förderbereichen moderiert bzw. durchgeführt; dabei dominieren Förderungen der regionalen Entwicklung, Unternehmensförderung und Infrastrukturmaßnahmen, Kultur- und Jugendförderung, berufliche Qualifizierungen sowie Integrationsmaßnahmen. Aktive Projektträger oder Projektpartner waren in Oldenburg auch Unternehmen oder wirtschaftsnahe Einrichtungen (z. B. Kammern, vgl. Tab. 2).

Die Tabelle 2 zeigt, dass die von Hochschulen und Forschungseinrichtungen durchgeführten EU-Projekte im Durchschnitt das höchste Fördervolumen besaßen (ca. $223000 €$ ). Die EU-Projekte, die von der Stadt Oldenburg oder sozialen Vereinigungen und Einrichtungen (insbesondere Kirchen und Wohlfahrtsverbänden) in Oldenburg durchgeführt wurden, verzeichneten durchschnittliche Fördersummen in der Größenordnung von etwa $170000 €$. Kleinstprojekte wurden insbesondere an den Schulen durchgeführt. Diese EUProjekte wurden im Mittel mit $4000 €$ (überwiegend aus dem COMENIUS-Programm) unterstützt.

Hebt man nun auf die Bedeutung der Kommune für die Akquirierung von EU-Mitteln ab, so fällt ihr Beitrag zunächst einmal eher sparsam aus. Lediglich 24 der 210 EU-Projekte im Zeitraum 1997-2002 fanden in Trägerschaft der Stadt Oldenburg statt oder hatten die Stadt Oldenburg als Projektpartner. Das entspricht einem Anteil von etwa $12 \%$. Werden die Finanzvolumina der Projekte zugrunde gelegt, so schneidet die Stadt Oldenburg etwas besser ab. Von den zwischen 1997 und 2002 insgesamt verausgabten Fördermitteln in Höhe von 24,5 Mio. $€$ entfielen auf EU-Projekte, an der die Stadt Oldenburg als Träger oder Partner beteiligt war, etwa $16 \%(3,9$ Mio. $€)$. De facto war jedoch das Engagement der Stadt Oldenburg höher. Nimmt man nämlich diejenigen EU-Projekte hinzu, an denen die Stadt Oldenburg beratend, informierend oder als Finanzpartner beteiligt war, so beläuft sich die Anzahl auf mindestens $41 \mathrm{EU}$-Projekte (und damit auf einen Anteil von knapp $20 \%$ ). Der Stadt Oldenburg kommt also, jenseits einer eigenen Projektträger- oder Projektpartnerschaft, eine wichtige Rolle bei der Initiierung und Vermittlung von EU-Fördermitteln zu (z. B. in Kooperation mit Unternehmen oder sozialen oder wirtschaftsnahen Einrichtungen und Institutionen). Über ebenfalls sehr gute Möglichkeiten der EU-Mittelakquirierung scheinen auch die Hochschulen und For- 
schungseinrichtungen zu verfügen. Sie schöpfen EUFördermittel in erheblichem Umfang ab. Offenbar werden die Fördermöglichkeiten an Hochschulen und Forschungseinrichtungen sehr genau ausgewertet und diese Kenntnisse und Informationen auch weitergegeben. Die übrigen Projektträger oder Projektpartner treten bedeutend seltener in Erscheinung. Bezogen auf diese Gruppen wäre zu fragen, ob die sozialen, ökonomischen oder kulturellen Akteure sowie die Qualifizierungsträger bei einer umfassenderen oder gezielteren Beratung oder Unterstützung noch mehr EU-Fördermittel hätten erschließen können.

\subsection{Qualitative Analysen von sieben Oldenburger EU-Projekten}

In diesem zweiten Untersuchungsfeld interessierte unter anderem, wie die EU-Förderprojekte auf kommunaler Ebene umgesetzt werden, inwieweit die kommunale Wirtschaftsförderung und kommunale Beratungsunternehmen ihrer Informations- und Moderatorenfunktion gerecht geworden sind oder welche Netzwerkstrukturen genutzt oder aufgebaut werden konnten. Um diese Untersuchungsziele zu erreichen, wurden sowohl einschlägige Projektdokumente und Projektinformationen ausführlich analysiert als auch ca. 30 qualitative Experteninterviews mit Projektleitern, Projektverantwortlichen in den betreuenden Institutionen sowie mit Projektteilnehmern geführt. Bei der Auswahl der sieben EU-Projekte wurde auf eine möglichst hohe inhaltliche wie organisatorische Spannbreite der Projektinhalte Wert gelegt (vgl. Tab. 3). Es wurde also eine "kontrastive Stichprobe“ gezogen. Zudem waren im Sample insbesondere solche EU-Projekte enthalten, in denen städtische Dienststellen als Projektbeteiligte im Spiel waren. Die Expertengespräche mit den Projektleitern und den Projektbeteiligten wurden transkribiert und inhaltsanalytisch ausgewertet. Die Aussagen der Experten konnten auf dieser Basis nach Themenfeldern segmentiert und entsprechenden analytischen Kategorien zugeordnet werden. Drei dieser Analysekategorien sollen hier explizit vorgestellt werden. Sie geben Aufschluss darüber, wie einerseits die inhaltlich-organisatorischen Anforderungen der EU-Förderpolitik und andererseits die kommunalen und behördlichen Beratungs- und Unterstützungsleistungen von Seiten der Projektakteure vor Ort wahrgenommen und bewertet werden. Auch der Aspekt der Vernetzung der regionalen Akteure spielte in den Interviews eine bedeutsame Rolle.

Tabelle 3

Ausgewählte EU-Projekte in der Stadt Oldenburg

\begin{tabular}{|c|c|c|}
\hline Projekttitel und -inhalt, EU-Programm & Projektbeteiligte aus Oldenburg & $\begin{array}{l}\text { Fördersumme } \\
\text { (Laufzeit) }\end{array}$ \\
\hline $\begin{array}{l}\text { Vernetzung und Stärkung kleiner und mittlerer } \\
\left.\text { Unternehmen der Automobilzuliefererbranche ( } 5 . \mathrm{FRP}^{1}\right)\end{array}$ & $\begin{array}{l}\text { Stadt Oldenburg, Unternehmen der } \\
\text { Automobilzuliefererbranche }\end{array}$ & $\begin{array}{l}454000 € \\
(1999 \text { und } 2000)\end{array}$ \\
\hline $\begin{array}{l}\text { Gewerbeflächenerschließung auf ehemaligen Militärflächen } \\
\text { (KONVER) }\end{array}$ & Stadt Oldenburg & $\begin{array}{c}1,1 \text { Mio. } € \\
(1997 \text { bis } 1999)\end{array}$ \\
\hline $\begin{array}{l}\text { Erweiterung und Umbau von zwei Kultureinrichtungen } \\
\text { im Oldenburger Bahnhofsviertel (EFRE/Ziel 2) }\end{array}$ & 2 Kulturspielstätten in Oldenburg & $\begin{array}{l}330000 € \\
(2001 \text { und } 2002)\end{array}$ \\
\hline Existenzgründungsagentur für Frauen (NOW) & $\begin{array}{l}\text { Existenzgründungsagentur für Frauen (EFA), } \\
\text { Oldenburg }\end{array}$ & $\begin{array}{l}310000 € \\
(2001 \text { und } 2002)\end{array}$ \\
\hline $\begin{array}{l}\text { Einzelbetriebliche Förderung von kleinen und mittleren } \\
\text { Unternehmen bei Erweiterungsvorhaben (EFRE/Ziel 2) }\end{array}$ & $\begin{array}{l}\text { Unternehmen des produzierenden Gewerbes, } \\
\text { Stadt Oldenburg }\end{array}$ & $\begin{array}{c}715000 € \\
(2000 \text { bis } 2006)\end{array}$ \\
\hline $\begin{array}{l}\text { Kooperation und Mobilität zwischen europäischen } \\
\text { Hochschulen (ERASMUS) }\end{array}$ & Universität Oldenburg & $\begin{array}{c}400000 € \\
(1997 \text { bis } 2002)\end{array}$ \\
\hline $\begin{array}{l}\text { Berufliche Integration von Migranten und Flüchtlingen } \\
\text { in Niedersachsen (INTEGRA) }\end{array}$ & $\begin{array}{l}\text { Zentrum für wiss. Zusammenarbeit, Universität } \\
\text { Oldenburg }\end{array}$ & $\begin{array}{c}\text { 2,8 Mio. } €^{2} \\
(1998 \text { bis } 1999)\end{array}$ \\
\hline
\end{tabular}

FRP: Forschungsrahmenprogramm

Fördersumme für den Regierungsbezirk Weser-Ems

Quelle: Carrefour Niedersachsen/Weser-Ems 2002 und eigene Erhebungen 


\section{Anforderungen an Zeit- und Humanressourcen der Projektakteure}

Die mit der Beantragung, Durchführung und Abwicklung von EU-Projekten verbundenen zeitlichen und organisatorischen Belastungen durch Verwaltungsaufwand und Behördenkontakte sind von vielen Projektbeteiligten thematisiert worden. Mehrfach wurde Kritik an den komplexen, formal-bürokratischen Anforderungen und Kontrollauflagen geäußert, die mit der EU-Mittelvergabe verbunden sind. Allerdings fallen in den untersuchten Projekten die Einschätzungen im Hinblick auf die Anforderungen an die Zeit- und Humanressourcen sehr unterschiedlich aus: Die hohe zeitliche und organisatorisch-bürokratische Belastung wird von Unternehmen sehr deutlich kritisiert. Der Aufwand für Projektbeantragung und Projektmanagement sei zusätzlich zum unternehmerischen Kerngeschäft zu erledigen. Hier würden oft die Grenzen der Belastbarkeit erreicht. Von Vertretern öffentlicher oder halböffentlicher Institutionen (z. B. Wirtschaftsförderung, Forschungseinrichtungen) oder sozialer Einrichtungen (z.B. Weiterbildungs- und Ausbildungsträger) wird der Verwaltungsaufwand nicht als zu hoch eingeschätzt. Die Beantragung von Fördermitteln ist für sie vielfach Routine und damit ein Teil ihres „Kerngeschäfts“.

\section{Beurteilung kommunaler Beratungsdienstleistungen}

Die Akteure in den untersuchten EU-Projekten konnten bei verschiedenen lokalen Stellen Beratungsdienstleistungen abfragen: bei der städtischen Wirtschaftsförderung, der Bezirksregierung Weser-Ems in Oldenburg oder dem Carrefour Niedersachsen/WeserEms (der von einem Consultingunternehmen geführt wird). Auch in diesem Punkt unterschieden sich die Auffassungen der Projektakteure entlang der oben skizzierten Linie. Eine Optimierung der Beratungsdienstleistung ist insbesondere nach Auffassung der befragten Projektbeteiligten in Unternehmen erforderlich. Sie forderten eine bessere Unterstützung bei der Erfüllung der formal-bürokratischen Anforderungen und damit eine zeitliche Entlastung. Von den Akteuren in anderen Projekten wurde dagegen kaum Kritik an den Beratungsleistungen geäußert. Im Gegenteil: Sie erhielten nach eigenen Angaben nützliche Hinweise oder lobten die faire und unproblematische Mittelbeantragung. Es werden zwei grundsätzlich unterschiedliche Erwartungsprofile deutlich. Die Anforderungen an Qualität und Quantität der kommunalen Beratungs- und Informationsleistungen sind also sehr heterogen.

\section{Etablierung von Netzwerkstrukturen und Synergieeffekte}

Aus der Sicht der befragten Leiter und Koordinatoren der untersuchten EU-Projekte konnten in einigen Fällen zwischen den beteiligten Akteuren in und um Oldenburg funktionierende Netzwerk- und Kooperationsbeziehungen etabliert und positive Entwicklungsimpulse gesetzt werden. Dabei wurde besonders hervorgehoben: ein verbesserter Informationsaustausch, kürzere Kommunikationswege, schnellere Verbreitung von Innovationen oder positive Synergieeffekte durch gemeinsame und abgestimmte Aktivitäten. Von positiven Erfahrungen durch Informationsaustausch und Netzwerkaufbau berichten den Untersuchungen zufolge insbesondere Projektleiter aus öffentlichen oder halböffentlichen Institutionen, $z$. B. aus der Arbeitsverwaltung und Qualifizierungsbranche. Bei ökonomischen Akteuren in Oldenburg, insbesondere wenn sie in Konkurrenzbeziehungen zueinander stehen, wurden deutlich seltener Netzwerkbildungen beobachtet. Ihr zentraler Ansprech- und Kooperationspartner waren die kommunalen Beratungsdienstleister (Wirtschaftsförderung, Consultingunternehmen), teilweise die wirtschaftsnahen Verbände.

\section{Erweiterung kommunaler Handlungs- spielräume durch EU-Regionalpolitik - Ergebniszusammenfassung und Schlussfolgerungen}

Erweitert die EU-Regionalpolitik kommunale Handlungsspielräume? Die Eingangsfrage der Studie ist für die Stadt Oldenburg relativ klar zu beantworten. Die Stadt Oldenburg war einer der aktiven Projektträger und Projektpartner. Zahlreiche Projekte wurden in kommunaler Verantwortung durchgeführt oder von der Stadt initiiert. Allein für Projekte aus den Bereichen Regionalentwicklung, Unternehmens-, Infrastrukturund Kulturförderung flossen im Beobachtungszeitraum mehr als 4 Mio. $€$ nach Oldenburg. Mit dieser Finanzspritze konnten Vorhaben realisiert werden, die sonst aus dem kommunalen Haushalt hätten finanziert werden müssen oder gar nicht umgeset $z t$ worden wären. Allerdings waren nicht nur städtische Behörden bei der Akquirierung von EU-Mitteln aktiv. Insbesondere Hochschulen und Forschungseinrichtungen verfügen offenbar über reichhaltige Erfahrungen bei der erfolgreichen Beantragung von Fördermitteln. Auch soziale Akteure und Einrichtungen konnten in größerem Umfang EU-Fördermittel einwerben.

Die Kommune bzw. die Stadt Oldenburg hat im Bereich der kommunalen EU-Politik eine doppelte Funktion: Einerseits beantragt sie selbst EU-Mittel und führt ent- 
sprechende Entwicklungsprojekte durch. Dafür muss in der Wirtschaftsförderung oder anderen kommunalen Dienststellen hinreichend qualifiziertes Personal zur Verfügung stehen. Andererseits nimmt sie eine Beratungs- oder Moderatorenfunktion für andere Antragsteller und Informationssuchende ein. Die qualitative Analyse zeigt, dass die Anforderungen an die Beratungsfunktion von Kommunen (oder beratenden Consultingunternehmen) sehr heterogen sind. Viele ökonomische, soziale oder kulturelle Akteure und Aktivitäten in der Kommune wären potenziell förderfähig. Allerdings sind die Kenntnisse und Fähigkeiten im Hinblick auf die EU-Antrags- und EU-Fördermodalitäten bei den Projektbeteiligten äußerst unterschiedlich. Einige legen eine sehr hohe Routine und Professionalität bei der EU-Mittel-Akquirierung und dem Projektmanagement an den Tag. Dies erfordert von den Beratungsdienstleistern eine entsprechend kompetente, kreative und zielorientierte Beratung und Unterstützung. Andere wiederum erwarten von den kommunalen Beratungsinstitutionen eine intensive Betreuung und zeitliche wie organisatorische Entlastung.

Die empirischen Ergebnisse deuten an einigen Stellen an, dass die EU-Mittelakquirierung und die Projektdurchführung Netzwerke zwischen Projektbeteiligten etablieren und in gewissem Rahmen kreative Milieus (Informationsaustausch, Nutzung von Synergien,...) fördern. Diese Netzwerkstrukturen entstanden insbesondere in solchen EU-Projekten, in denen soziale Einrichtungen (z.B. Vereine, Verbände, karitative Akteure), öffentliche Träger (u. a. Stadt, Bezirksregierung, Hochschulen) oder Qualifizierungsakteure (z.B. Arbeitsamt, Existenzgründungsagenturen, Weiterbildungsträger) miteinander kooperierten. Kontakte von Unternehmen untereinander konnten hingegen kaum etabliert werden. Eine „kommunale EU-Politik“ kann also durchaus auch Netzwerkstrukturen fördern. In einigen Bereichen sind diesbezüglich in Oldenburg sichtbare Erfolge erzielt worden. Der Aufbau von Unternehmensnetzwerken gestaltet sich aber vergleichsweise schwierig.

Abschließend noch einige Überlegungen zu der Frage, inwieweit die Oldenburger Ergebnisse auf andere deutsche Kommunen übertragbar sind: Sicherlich ist es nicht möglich, im Sinne eines Rankings die Stadt Oldenburg und die Effektivität ihrer kommunalen EUPolitik einzuordnen. Es dürfte allerdings deutlich geworden sein, dass ein Engagement in den EU-Förderprogrammen die kommunalen Gestaltungsspielräume insbesondere im Bereich der Stadt- und Regionalentwicklung sowie der Wirtschafts- und Kulturförderung erweitert. Auch Qualifizierungs- und Integrationsmaß- nahmen können aus EU-Mitteln (ko)finanziert werden und reduzieren somit die kommunalen Sozialausgaben. Betrachtet man die absoluten Größenordnungen der Förderbeträge, so amortisiert sich entsprechend qualifiziertes Personal (z. B. in kommunalen EU-Büros oder EU-Koordinierungsstellen) relativ rasch. Auch die Ergebnisse zu den kommunalen Beratungsdienstleistungen dürften auf andere Kommunen übertragbar sein. Kommunale EU-Beratung muss ein differenziertes und auf sehr unterschiedliche Zielgruppen ausgerichtetes Beratungsangebot bereithalten. Auch damit eröffnen sich die Kommunen wesentliche Gestaltungsspielräume.

\section{Anmerkung}

* Von den 215 EU-Projekten konnten fünf nicht eindeutig zugeordnet werden. Dementsprechend berücksichtigen die nachfolgenden Auswertungen nur 210 Projekte.

\section{Literatur}

Adamaschek, B. (2001): Der interkommunale Leistungsvergleich. Eine geeignete Strategie für Regionen? In: Informationen zur Raumentwicklung, H. 8, S. 503-509

Aschauer, W. (2000): Zwischen Analyse und Politik. Zum Anwendungsbezug regionalwissenschaftlicher Forschung. In: Informationen zur Raumentwicklung, H. 9/10, S. 589-598

Bathelt, H.; Glückner, J. (2000): Netzwerke, Lernen und evolutionäre Regionalentwicklung. In: Zeitschrift für Wirtschaftsgeographie, H. 3-4, S. 167-182

Butzin, B. (2000): Netzwerke, kreative Milieus und Lernende Region. In: Zeitschrift für Wirtschaftsgeographie, H. 3-4, S. $149-166$

Carrefour Niedersachsen/Weser-Ems (2002): EU-Projekte in Oldenburg. In: Stadt Oldenburg, Universität Oldenburg, Carrefour Niedersachsen/Weser-Ems (Hrsg.): Sternfänger. Europa in Oldenburg - Frfolgsgeschichten und Chancen. Oldenburg, S. $12-14$ und $34-42$

Danielzyk, R.; Oßenbrügge, J. (1996): Lokale Handlungsspielräume zur Gestaltung internationalisierter Wirtschaftsräume. Raumentwicklung zwischen Globalisierung und Regionalisierung. In: Zeitschrift für Wirtschaftsgeographie, H. 1-2, S. $101-112$

Eser, T. W. (2001): Evaluation und Qualitätsmanagement. Anforderungen und Konsequenzen für die EU-Strukturpolitik. In: Informationen zur Raumentwicklung, H. 6/7, S. 327-339

Grabow, B.; Henckel, D. (1999): Kommunale Wirtschaftspolitik. In: Wollmann, H.; Roth, R. (Hrsg.): Kommunalpolitik. Politisches Handeln in den Gemeinden. Opladen: Leske und Budrich, S. 616-632 
Foißner, P. (2000): Endogene Entwicklungen in peripheren Regionen. Möglichkeiten der Aktivierung endogener Potenziale in der Region Vorpommern. In: Raumforschung und Raumordnung, H. 4, S. 297-306

Icks, A,; Richter, M. (1999): Kommunale Wirtschaftsförderung. Ein innovatives Modell. In: Standort. Zeitschrift für Angewandte Geographie, H. 4, S. 9-14

Langer, M. (2002): Oldenburg. Eine Stadt mitten in Europa. In Stadt Oldenburg, Universität Oldenburg, Carrefour Niedersachsen/Weser-Ems (Hrsg.): Sternfänger. Europa in Oldenburg - Erfolgsgeschichten und Chancen. Oldenburg, S. 7-8

Möller, J.; Tassinopoulos, A. (2000): Zunehmende Spezialisierung oder Strukturkonvergenz? Fine Analyse der sektoralen Beschäftigungsentwicklung auf regionaler Ebene. In: Jahrbuch für Regionalwissenschaft, Nr. 20, S. 1-38

Nutzenberger, K. M. (2002): Weiterentwicklung auf sicherer Grundlage. Das neue Europabüro des DStGB in Brüssel. In: Stadt und Gemeinde interaktiv. Themenheft Kommunen und Europa, H. 6, S. 223-224

Rösch, A. (2000): Kreative Milieus als Faktoren der Regionalentwicklung. In: Raumforschung und Raumordnung, H. 2-3, S. $163-172$

Rolfes, M. (2002): Oldenburger EU-Projekte auf dem Prüfstand. In: Stadt Oldenburg, Universität Oldenburg, Carrefour Niedersachsen/Weser-Ems (Hrsg.): Sternfänger. Europa in Oldenburg - Erfolgsgeschichten und Chancen. Oldenburg, S. 15-18

Schnappauf, W. (1999): Die Organisation der Kreisverwaltung und deren Reform/Modernisierung. In: Wollmann, H.; Roth, $\mathrm{R}$. (Hrsg.): Kommunalpolitik. Politisches Handeln in den Gemeinden. Opladen: Leske und Budrich, S. 430-436
Stucke, N.; Schöneich, M. (1999): Organisation der Stadtverwaltung und deren Reform/Modernisierung. In: Wollmann, H.; Roth, R. (Hrsg.): Kommunalpolitik. Politisches Itandeln in den Gemeinden. Opladen: Leske und Budrich, S. 411-429

Stüber, M. (2002): Europäische Förderprogramme. Zwischen Ansporn, Anspruch und Aufwand. In: Stadt Oldenburg, Universität Oldenburg, Carrefour Niedersachsen/Weser-Ems (Hrsg.): Sternfänger. Europa in Oldenburg - Erfolgsgeschichten und Chancen. Oldenburg, S. 5-6

Thömmes, C.; Saller, R. (2002): München in Europa. Europa der Landeshauptstadt 2001/2002. München: Referat für Arbeit und Wirtschaft der Landeshauptstadt München. = Veröffentlichungen des Referates für Arbeit und Wirtschaft, H. 134 (April 2002)

Thränhardt, D. (1999): Die Kommunen und die Europäische Union. In: Wollmann, H.; Roth, R. (Hrsg.): Kommunalpolitik. Politisches Handeln in den Gemeinden. Opladen: Leske und Budrich, S. 361-377

Toepel, K. (2000): Evaluation in der Regionalpolitik. In: Deutsches Institut für Wirtschaftsforschung (Hrsg.): Vierteljahreshefte zur Wirtschaftsforschung, H. 3, S. 395-405

Wollmann, H. (2000): Evaluierung und Evaluierungsforschung von Verwaltungspolitik und -modernisierung - zwischen Analysepotenzial und -defizit. In: Stockmann, R. (Hrsg.): Evaluationsforschung. Grundlagen und ausgewählte rorschungsfelder. Opladen: Leske und Budrich, S. 195-231

Dr. Manfred Rolfes Universität Osnabrück Fachgebiet Geographie Seminarstraße $19 \mathrm{a} / \mathrm{b}$ 49069 Osnabrück E-Mail: Mrolfes@uos.de 